\title{
Interacting polariton fluids in a monolayer of tungsten disulfide
}

\author{
Fábio Barachati, ${ }^{1}$ Antonio Fieramosca, ${ }^{2}$ Soroush Hafezian, ${ }^{1}$ Jie Gu, ${ }^{3}$ Biswanath Chakraborty,${ }^{3}$ Dario \\ Ballarini, ${ }^{2,}$ 米 Ludvik Martinu, ${ }^{1}$ Vinod Menon, ${ }^{3}$ Daniele Sanvitto, ${ }^{2}$ and Stéphane Kéna-Cohen ${ }^{1, \dagger}$ \\ ${ }^{1}$ Department of Engineering Physics, École Polytechnique de Montréal, Montréal H3C 3A7, QC, Canada \\ ${ }^{2}$ CNR - NANOTEC, Istituto di Nanotecnologia, Lecce 73100, Italy \\ ${ }^{3}$ Department of Physics, City University of New York, New York 10031, NY, USA
}

(Dated: March 14, 2018)

Keywords: Bloch surface waves, strong coupling, exciton-polaritons, two-dimensional materials, transition metal dichalcogenides, tungsten disulfide.

Atomically thin transition metal dichalcogenides (TMDs) possess a number of properties that make them attractive for realizing roomtemperature polariton devices ${ }^{1}$. An ideal platform for manipulating polariton fluids within monolayer TMDs is that of Bloch surface waves, which confine the electric field to a small volume near the surface of a dielectric mirror ${ }^{2 / 5}$. Here we demonstrate that monolayer tungsten disulfide $\left(\mathrm{WS}_{2}\right)$ can sustain Bloch surface wave polaritons (BSWPs) with a Rabi splitting of $43 \mathrm{meV}$ and propagation constants reaching $33 \mu \mathrm{m}$. In addition, we evidence strong polariton-polariton nonlinearities within BSWPs, which manifest themselves as a reversible blueshift of the lower polariton resonance by up to $\mathbf{1 2 . 9} \pm \mathbf{0 . 5} \mathrm{meV}$. Such nonlinearities are at the heart of polariton devices 6 and have not yet been demonstrated in TMD polaritons. As a proof of concept, we use the nonlinearity to implement a nonlinear polariton source. Our results demonstrate that BSWPs using TMDs can support long-range propagation combined with strong nonlinearities, enabling potential applications in integrated optical processing and polaritonic circuits.

Strong light-matter coupling with TMD excitons has previously been demonstrated in a variety of systems, including planar microcavities $\frac{13}{17}$ and plasmonic cavities16118/19. At room-temperature, however, these structures have been limited to extremely short polariton lifetimes due to the high losses of the underlying cavity and the broad exciton linewidth. Here, we overcome these limitations by strongly coupling the A exciton of monolayer $\mathrm{WS}_{2}$ to a low-loss propagating Bloch surface wave at the air-dielectric interface of a Bragg mirror. Tungsten disulfide was chosen as the active material over other TMDs due to its strong and sharp excitonic absorption, which better matches the narrow linewidths of Bloch surface modes. The low losses of the dielectrics and the thin monolayer enable polaritons to propagate over the entire extent of the monolayer. To highlight the role of polariton-polariton nonlinearities, we demonstrate a nonlinear polariton source in a configuration analogous to that previously used to show bistability under continuous wave excitation 20 . All measurements reported here were performed under ambient conditions.

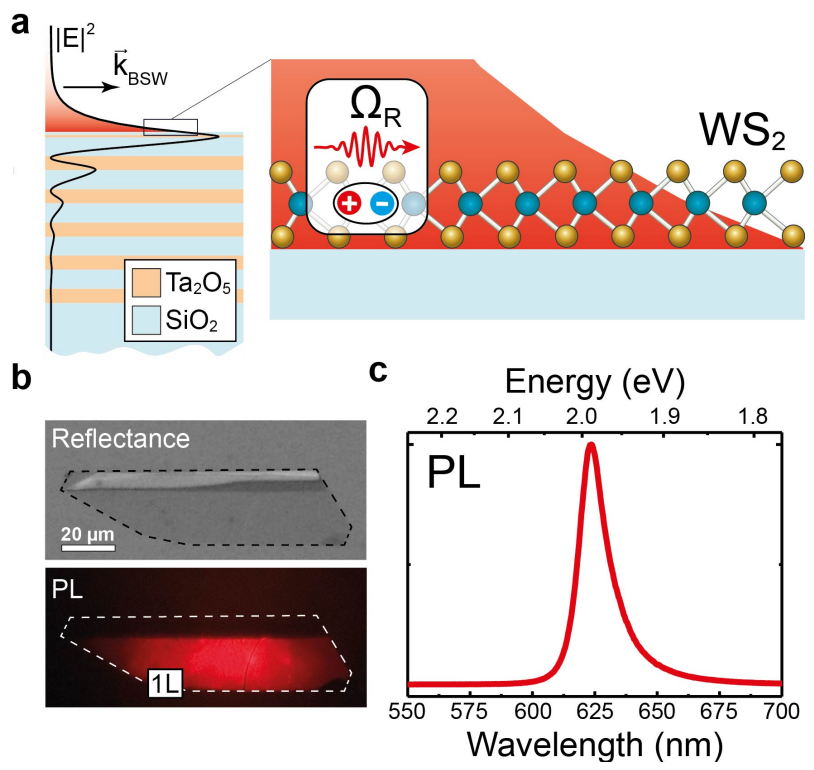

Figure 1 Sample structure and monolayer characterization. (a) Schematic of the dielectric stack supporting Bloch surface wave polaritons in monolayer $\mathrm{WS}_{2}$. The solid black lines trace the electric field profile of the bare mode at the wavelength of the A exciton band. The mode is TE polarized and propagates along the surface with wavevector $\vec{k}_{B S W}$. The inset illustrates the coupling of the enhanced electric field at the surface of the stack to the in-plane excitons in the monolayer. (b) Micrographs of the monolayer in reflectance and PL. The dashed lines indicate the flake boundaries. Only monolayer regions show bright PL under illumination by a large Gaussian spot. (c) The typical monolayer PL spectrum under $514 \mathrm{~nm}$ excitation contained a single strong peak centered at $1.988 \mathrm{eV} / 623.6$ $\mathrm{nm}$.

A schematic of our sample is shown in Fig. 11. A glass coverslip was coated with a dielectric mirror, designed to support a Bloch surface mode near the A exciton band of $\mathrm{WS}_{2}(2.014 \mathrm{eV} / 615.6 \mathrm{~nm})^{21}$. A large monolayer of $\mathrm{WS}_{2}$ was then transferred onto the top dielectric surface. The solid line in Fig. 19 shows the calculated electric field profile corresponding to the bare Bloch mode at the A exciton wavelength. The field peaks inside the last dielectric pair and decays exponentially away from 

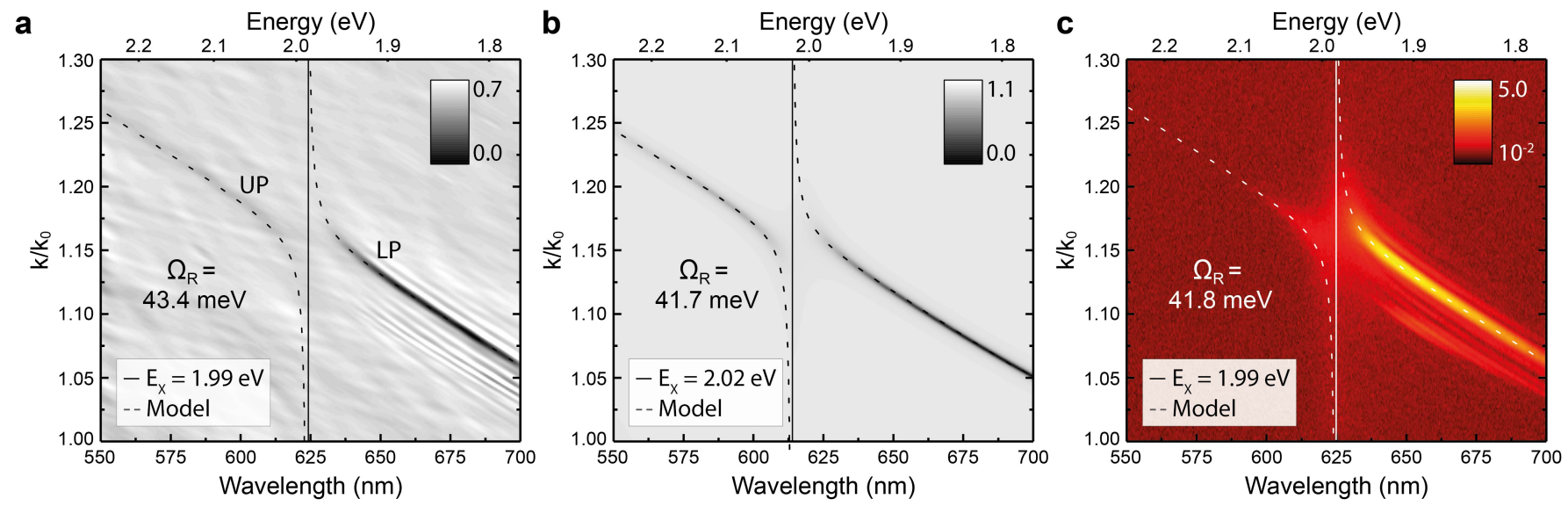

Figure 2 Bloch surface wave polaritons. (a) Experimental dispersion of the Bloch surface wave polaritons in a monolayer $\mathrm{WS}_{2}$ in reflectance and (b) the corresponding transfer matrix calculation. The experimental and theoretical dispersions for the bare Bloch mode are shown in Fig. S1. (c) Experimental dispersion in PL under $514 \mathrm{~nm}$ excitation on a logarithmic color scale. Coupled harmonic oscillator fits and fitting parameters are shown in all three panels. The UP and LP modes are traced in dashed lines and the fitted exciton energies are indicated by solid lines.

the surface. The mode is TE polarized and propagates along the surface with wavevector $\vec{k}_{B S W}$. Fig. 1 p shows a micrograph of the large exfoliated $\mathrm{WS}_{2}$ flake in reflectance (top) and in photoluminescence (PL, bottom) under $514 \mathrm{~nm}$ excitation by a large Gaussian spot. Only the monolayer regions exhibit bright PL due to their direct bandgap22. A typical monolayer PL spectrum is shown in Fig. 11 and contains a single strong peak centered at $1.988 \mathrm{eV} / 623.6 \mathrm{~nm}$ with a FWHM of $42 \mathrm{meV}$. These values vary slightly along the sample, presumably due to fluctuations in strain, substrate adhesion, defects and surface charge density 23124 .

To demonstrate strong coupling between the monolayer A exciton and the Bloch surface mode, white-light reflectivity was measured with an immersion objective in a back focal plane (BFP) imaging configuration (see Methods). In Fig. 2a we show the experimental dispersion of the upper (UP) and lower (LP) polariton modes measured in the center of the monolayer. The position and visibility of the modes are in good agreement with transfer matrix calculations shown in Fig. 2 $\mathrm{b}$, where the thickness and refractive index of monolayer $\mathrm{WS}_{2}$ were obtained from the literature ${ }^{21}$. Both polariton branches were also visible in PL, as shown in Fig. 2p on a logarithmic color scale. Their characteristic anti-crossing is visible in both reflectance and PL around the same wavelength of $623 \mathrm{~nm}$, coinciding with the peak PL wavelength shown in Fig 1 1 . Interestingly, a progression of modes surrounding the LP branch is visible in Fig. 2 a,c. Bloch surface waves are extremely sensitive to changes in the thickness and refractive index of the topmost layer. In the case of our monolayer, these can be caused by surface inhomogeneities in the large area probed by the propagating mode. A similar but weaker effect can also be seen for the bare mode, shown in Fig. S1a. For each panel in Fig. 2, a simple $2 \times 2$ coupled harmonic oscilla- tor $(\mathrm{CHO})$ model was used to fit the data (see Methods). The dispersion fits and exciton energies are traced in dashed and solid lines, respectively. The extracted Rabi splittings of $43.4 \pm 0.8 \mathrm{meV}$ and $41.8 \pm 0.6 \mathrm{meV}$ are in close agreement with the transfer matrix value of $41.7 \pm$ $0.3 \mathrm{meV}$.

Next, we investigated how far BSWPs are able to propagate within the $\mathrm{WS}_{2}$ monolayer. The pump wavevector and wavelength were selected to be in resonance with the LP mode. The first panel in Fig. 33 shows the real space propagation trace for an exciton fraction of $10 \%$ and wavelength of $645 \mathrm{~nm}$. Propagation can be observed for over $60 \mu \mathrm{m}$ and ends upon reaching the flake boundary, indicated by a dashed white line. As a comparison, the propagation of the uncoupled (bare) Bloch surface wave is shown in panel 2. The propagation constants extracted from single exponential decay fits were $20.6 \pm 0.1 \mu \mathrm{m}$ and $21.1 \pm 0.1 \mu \mathrm{m}$ for the $\mathrm{LP}$ and bare modes, respectively. These values are considerably larger than the ones found in high-quality planar microcavities embedding TMD monolayers, which are typically of the order of $1 \mu \mathrm{m} \frac{14}{14}$ Here, the propagation length can be further increased by limiting the angular content of the excitation beam. By reducing divergence in this way, the propagation length of the bare mode could be increased to $42.2 \pm 0.2 \mu \mathrm{m}$ (Fig. S3). We observe that the presence of a small crack in the flake, indicated by a solid red line in panel 1 , has very little impact on the propagation, possibly due to the high photonic content of the mode. The third panel in Fig. 3a shows an enlarged micrograph of the monolayer in reflectance where the small crack and monolayer boundaries can be seen. Given that these measurements were performed using pulsed excitation, we illustrate in Figure $3 \mathrm{~b}$ the underlying temporal dynamics by calculating the spatial density of BSWPs under our experimental conditions (see Methods and the Supplementary Information). The temporal profile of the 

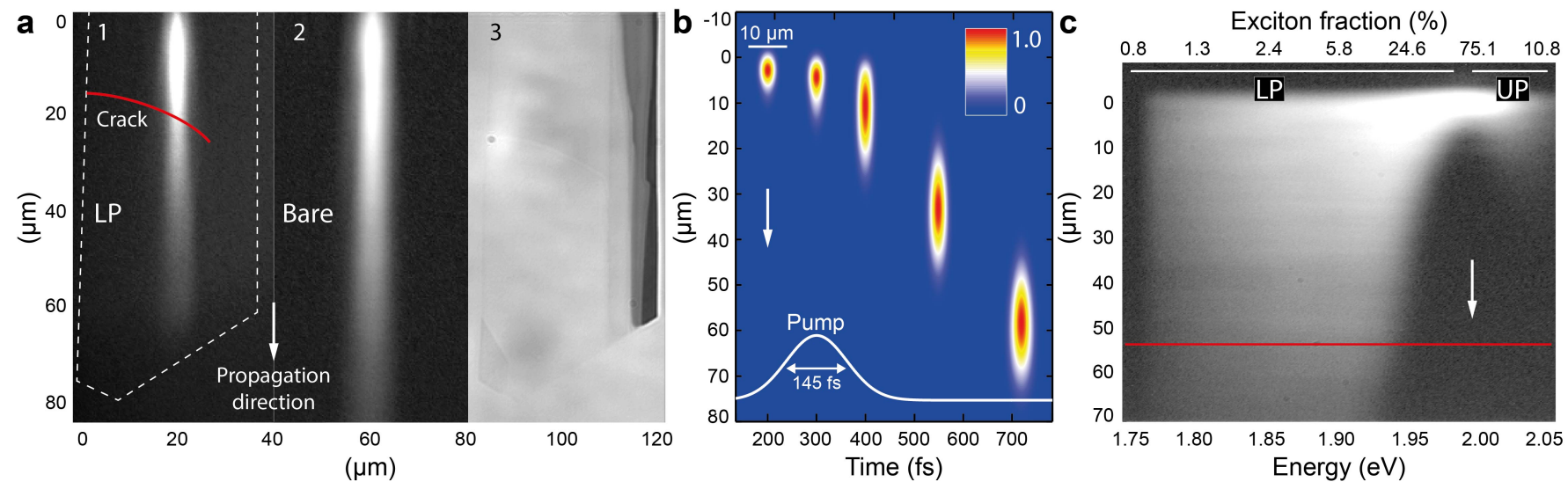

Figure 3 Polariton propagation. (a) Resonant propagation at a wavelength of $645 \mathrm{~nm}$ for the LP (exciton fraction of $10 \%$ ) and bare modes, shown in panels 1 and 2, respectively. Corresponding propagation constants are $20.6 \pm 0.1 \mu \mathrm{m}$ and $21.1 \pm 0.1 \mu \mathrm{m}$. The flake boundary is shown by a white dashed line and a small crack is indicated by a solid red line. Panel 3 shows a micrograph of the monolayer in reflectance where the crack and boundaries can be seen. (b) Calculated time snapshots of the spatial density of BSWPs following the arrival of a laser pulse, traced in a solid white line. The chirped pump pulse has an estimated FWHM of $145 \mathrm{fs}$. The polariton wavepacket is initially concentrated close to the excitation spot. As the pump vanishes, it can be seen propagating downwards at a group velocity of $1.49 \cdot 10^{8} \mathrm{~m} \mathrm{~s}^{-1}$. The propagation traces were displaced laterally so that the center of the excitation spot coincides with the time at which the snapshot was taken. The top scale bar is related to the spatial dimensions of the propagation traces in the horizontal direction.(c) Non resonant (PL) propagation under $514 \mathrm{~nm}$ excitation on a logarithmic intensity scale. The solid red line indicates the position of a small crack on the flake. Propagation constants for the LP branch ranged from $14.7 \pm 0.1 \mu \mathrm{m}$ to $33.4 \pm 0.4 \mu \mathrm{m}$ for exciton fractions between $15.7 \%$ to $1.7 \%$. Polaritons from the upper branch are also visible but propagate significantly less. In both measurements the propagation direction is downwards, as indicated by the white arrows.

pump is traced in a solid white line. During the pump pulse, the density of polaritons is highest close to the excitation spot, centered at $\mathbf{r}=0$. As the pump vanishes, the polariton wavepacket can be clearly seen as it propagates downwards with a group velocity of $1.49 \cdot 10^{8} \mathrm{~m} \mathrm{~s}^{-1}$.

Propagation was also investigated using non-resonant above-gap excitation. In this case, the pump first creates excitons, which subsequently relax into propagating polariton states. Fig. 3r shows the PL spectrum under $514 \mathrm{~nm}$ excitation as it propagates within the flake. The propagation constants for polaritons with exciton fractions ranging from $1.7 \%$ to $15.7 \%$ were found to be between $33.4 \pm 0.4 \mu \mathrm{m}$ and $14.7 \pm 0.1 \mu \mathrm{m}$, consistent with the resonant case. Polaritons from the upper branch are also visible in Fig. 3r due to the logarithmic intensity scale, but propagate significantly less. Again, the presence of the small crack is indicated by the solid red line and only a negligible effect was seen on the intensity profiles. The single-exponential fits for both resonant and non-resonant propagation traces are shown in Fig. S2.

The experimental LP propagation constant for an exciton fraction of $15.7 \%$ agrees well with the value of 15.3 $\mu \mathrm{m}$, estimated from the group velocity and a $6.4 \mathrm{meV}$ linewidth (103 fs lifetime), extracted from the reflectivity spectra. The maximum propagation constant that can be estimated from the dispersion relation is bound by our detection limit of $5 \mathrm{meV}$ to $19.6 \mu \mathrm{m}$. From the experimental propagation constants we obtain a linewidth of $2.4 \mathrm{meV}$ for the bare mode, corresponding to a quality factor $Q=800$ at a wavelength of $645 \mathrm{~nm}$.
Next, we study the effect of polariton-polariton interactions. At high densities, polaritons interact through their matter component due to phase space filling (PSF) and inter-particle Coulombic interactions, which under most conditions are repulsive and lead to a blueshift of the polariton modes. Fig. 4 a shows the resonant blueshift of the LP mode at the highest incident power density $\left(600 \mathrm{~W} \mathrm{~cm}^{-2}\right)$ as a function of the resonance position in the linear regime and the exciton fraction extracted from the CHO model shown in Fig. 2 a. In Fig. 40 we show the complete power sweep where the highest time-averaged blueshift of $12.9 \pm 0.5 \mathrm{meV}$ was observed. The top two curves for low and high powers show that the blueshift is reversible and is larger than the LP linewidth of $7 \sim 8$ $\mathrm{meV}$. The LP dispersions at low and high pump powers are shown in Fig. S4. The blueshift saturates with power (Fig. S5), as evidenced by flattening out of the dashed line in Fig. $4 \mathrm{~b}$ at higher powers. The saturation mechanism is likely due to a combination exciton-exciton annihilation and a possible dynamic transition to weak coupling at the highest powers 25 .

Estimating the polariton-polariton interaction constants from the experimental LP blueshift requires an accurate knowledge of the polariton density. In addition, when several modes in momentum space are excited, their individual contributions to the blueshift must be accounted for ${ }^{6}$. Using input-output theory and parameters corresponding to our experimental conditions, we have performed time-dependent calculations of the polariton density per mode in momentum space (see Methods and 

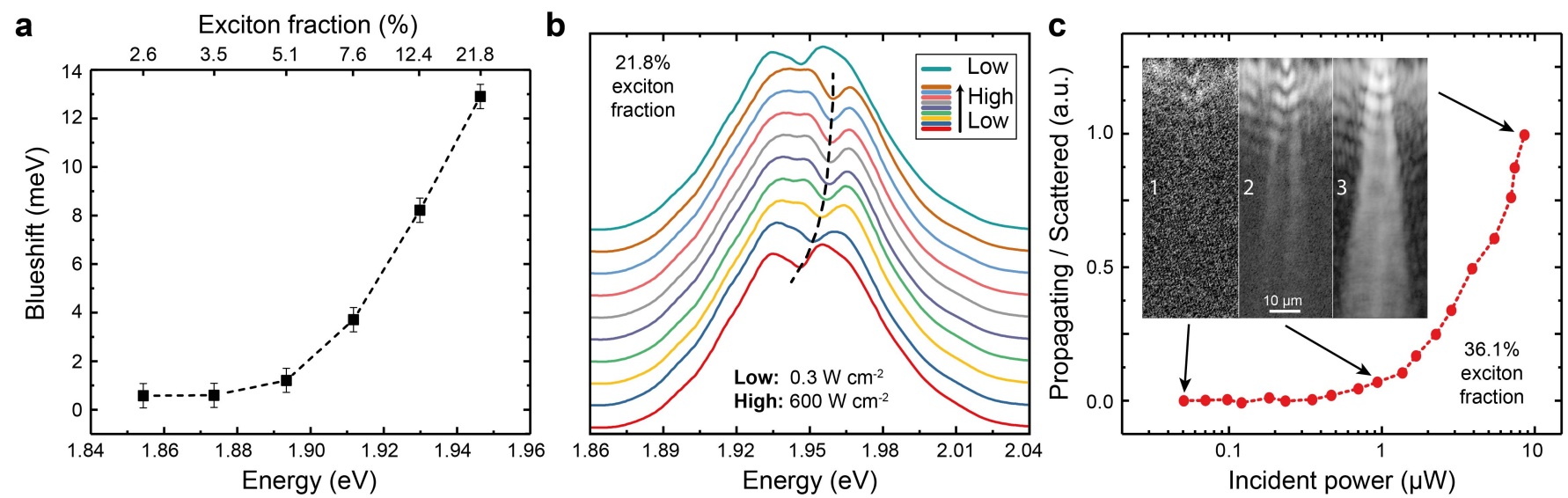

Figure 4 Nonlinear behaviour. (a) High power $\left(600 \mathrm{~W} \mathrm{~cm}^{-2}\right)$ time-averaged resonant blueshift of the LP mode as a function of the initial BSWP energy and corresponding exciton fraction, extracted from the CHO model shown in Fig. 22a. (b) Power sweep in steps of $75 \mathrm{~W} \mathrm{~cm}^{-2}$ for the highest blueshift/exciton fraction, showing that the shift is reversible and larger than the LP linewidth of 7 8 meV. (c) A nonlinear polariton source, where the incident power can block or launch a beam of propagating BSWPs. The red curve shows the normalized ratio of the propagating and scattered beams, which depend on their integrated areas, illustrating the nonlinear dependence on the incident pump power.

Fig. S6). Over the range of momenta probed, our experiments cannot determine the relative contribution of each interaction mechanism (Fig. S7). However, we can separately estimate the required exciton-exciton $\left(V_{X X}\right)$ and PSF $\left(V_{S A T}\right)$ interaction constants required to explain the observed blueshift. We find $V_{X X}=0.5 \pm 0.2 \mu \mathrm{eV} \mu \mathrm{m}^{2}$ and $V_{S A T}=0.09 \pm 0.04 \mu \mathrm{eV} \mu \mathrm{m}^{2}$. Remarkably, these values are considerably higher than those reported for other room-temperature systems $26 \mid 27$.

Our interaction constants can also be compared to theoretical values. The exciton-exciton interaction constant in TMDs can be estimated using $V_{X X} \simeq 2.07 E_{1 s} \lambda_{X}^{2}=$ $1.9 \mu \mathrm{eV} \mu \mathrm{m}^{2}$, where $E_{1 s}=0.32 \mathrm{eV}$ is the 1 s exciton binding energy and $\lambda_{X}=1.7 \mathrm{~nm}$ is the $1 \mathrm{~s}$ exciton radius 28 . The numerical prefactor in the expression for PSF depends on the form of the exciton wavefunctions. For 1s excitons, it is often estimated ${ }^{6}$ as $V_{S A T} \simeq 7.18 \hbar \Omega_{R} \lambda_{X}^{2}=$ $0.87 \mu \mathrm{eV} \mu \mathrm{m}^{2}$. Both theoretical values slightly exceed those obtained from the blueshift. This is, however, consistent with the fact that peak densities were used in our estimates, but experimental time-averaging will reduce the apparent blueshift. These estimates also suggest that both Coulombing interactions and PSF play a comparable role within the range of momenta probed in our system.

Finally, we demonstrate how the optical control of the LP mode can be used as a nonlinear source of polaritons. The pump bandwidth was limited to $3 \mathrm{~nm}$ centered at 633 $\mathrm{nm}$ and at this wavelength the LP resonance is too broad and shallow to be resolved in Fig. 2a. At low incident powers, very little light is coupled into the propagating polariton mode, as shown on inset 1 in Fig. 4k. As the pump power is increased, the LP mode blueshifts and the propagating part of the BSWP dispersion moves closer to resonance with the pump (insets 2 and 3). At high power, the pump laser is fully resonant with the polariton mode and launches a propagating surface wave, which through coupling to the underlying bare mode is able to propagate far beyond the flake dimensions. The normalized ratio of the propagating and scattered powers, which depends on the selected integration areas, is shown in the red curve, illustrating the nonlinear dependence on the incident pump power. This behaviour is analogous to the onset of bistability, which can be observed for continuous wave pumping in planar microcavities ${ }^{20}$.

Our demonstration of strong light-matter coupling between excitons in two-dimensional materials and propagating Bloch surface waves introduces an exciting new platform for the study of interacting polariton fluids at room temperature. Polariton propagation losses stem mainly from leakage into the immersion optics, which could be reduced by an increase in the number of dielectric pairs or even completely avoided by the use of surface gratings for external in- and out-coupling 29 . The simple dielectric structure can be tailored for other twodimensional materials or for multilayer heterostructures. In particular, TMDs encapsulated by hexagonal boron nitride show improved surface flatness, screening from charged impurities and ambient stability. The reduced disorder can lead to a suppression of loss mechanisms such as exciton-exciton annihilation and allow higher polariton densities to be achieved in the nonlinear regime ${ }^{30}$. Interacting BSWPs based on TMDs can enable the fabrication of room-temperature nonlinear polariton devices with long propagation distances. Together with properties such as high photostability, valley polarization and tunability by electric fields, TMDs could also bring new functionalities to polaritonic circuits. 


\section{METHODS}

The Bragg mirror consisted of five pairs of tantalum pentoxide $\left(\mathrm{Ta}_{2} \mathrm{O}_{5}\right) /$ silicon dioxide $\left(\mathrm{SiO}_{2}\right)$ layers $(98.5$ $\mathrm{nm} / 134.6 \mathrm{~nm}$ thick), deposited by radiofrequency magnetron sputtering at a pressure of $10^{-7}$ mbar. An additional thinner pair $(17.1 \mathrm{~nm} / 22.3 \mathrm{~nm})$ was used to shift the position of the Bloch mode at the tungsten disulfide $\left(\mathrm{WS}_{2}\right)$ A exciton wavelength towards the center of the photonic bandgap. A large monolayer of $\mathrm{WS}_{2}$ was first tape-exfoliated onto a polydimethylsiloxane stamp and subsequently transferred onto the top dielectric surface.

The experimental setup for reflectivity measurements consisted of an inverted microscope (Olympus IX-81) equipped with a 1.42 numerical aperture (NA) oil immersion objective. At the microscope's side port, three twoinch achromatic doublet lenses (Thorlabs, focal lengths $20 / 7.5 / 30 \mathrm{~cm}$ ) were used to project a magnified image of the back focal plane (BFP) onto the slit of an imaging spectrometer with a cooled CCD camera (Princeton Instruments, IsoPlane 160 spectrometer, PIXIS 400B eXcelon camera, $300 \mathrm{~g} / \mathrm{mm}$ grating with $500 \mathrm{~nm}$ blaze, $50 \mu \mathrm{m}$ slit). The white light source (Energetiq, EQ99X) was spatially filtered by a single-mode fiber (Thorlabs P1-630A-FC) and a large area Gaussian collimator (SLT, LB20). The same setup was used for photoluminescence (PL) and non-resonant propagation measurements by using a CW $514 \mathrm{~nm}$ diode laser (Thorlabs L520P50/LTC56B) as the light source, coupled to a Thorlabs P1-405B-FC fiber. The filters used were Omega Filters RPE520SP (excitation), Semrock FF538-FDi01 (dichroic) and Thorlabs FELH0550 (detection).

For resonant propagation and nonlinear measurements, a tunable femtosecond laser (estimated pulse width of $145 \mathrm{fs}$, repetition rate $10 \mathrm{kHz}$ ) was focused onto the BFP of a $1.49 \mathrm{NA}$ oil immersion objective with a long focal length lens $(75 \mathrm{~cm})$. A home built microscope was used with the same lenses as the setup above, except for a $20 \mathrm{~cm}$ tube lens. The energy and focusing position of the laser were adjusted to be resonant with the mode of interest and the corresponding real space spot size dimensions were typically $3 \mu \mathrm{m} \times 5 \mu \mathrm{m}$ (full-width at half-maximum). The same spectrometer was used. Both the bare Bloch surface wave and the Bloch surface wave polariton modes were visible in reflectance close to the boundaries of the monolayer due to the reduced coupling strength. During propagation, polaritons approaching the boundary leaked light into the underlying bare mode and appeared to propagate beyond the boundary of the flake with a small change in propagation direction. For propagation measurements, adjustable slits were placed in the BFP to select only the lower polariton (LP) mode and in the image plane to block the excitation spot (in the resonant case only).

Reflectivity and PL spectra were fitted with the simple
$2 \times 2$ coupled harmonic oscillator Hamiltonian

$$
\hat{H}_{\mathbf{k}}=\left(\begin{array}{cc}
E_{B S W}(\mathbf{k}) & \Omega_{R} / 2 \\
\Omega_{R} / 2 & E_{E X}
\end{array}\right),
$$

where $\Omega_{R}$ is the Rabi splitting, $E_{E X}$ is the exciton energy and $E_{B S W}(\mathbf{k})$ is the energy dispersion of the bare Bloch surface mode, which can be approximated by

$$
E_{B S W}(\mathbf{k})=\hbar v_{g}|\mathbf{k}|+E_{0}
$$

in the center of the photonic bandgap. Here, $v_{g}$ is the group velocity and $E_{0}$ is a fitting parameter.

For numerical simulations, we calculate the LP field in momentum space $\psi_{L P}(\mathbf{k}, t)$. Its time-evolution is governed by

$$
\begin{array}{r}
i \hbar \frac{\partial \psi_{L P}(\mathbf{k}, t)}{\partial t}=\left[E_{B S W P}(\mathbf{k})-\hbar \omega_{P}-\right. \\
\left.+\hbar \gamma_{L P}\right] \psi_{L P}(\mathbf{k}, t) \\
+\hbar P(\mathbf{k}, t), \quad(3)
\end{array}
$$

where $E_{B S W P}(\mathbf{k})$ is a linear approximation of the LP dispersion in the vicinity of the pump, $\hbar \omega_{P}$ is the pump energy, $\gamma_{L P}$ is the LP dissipation rate and $P(\mathbf{k}, t)$ is the driving term. To reproduce our experimental conditions, the pump field is taken to be a Gaussian in momentum space modulated by a positively chirped temporal Gaussian envelope.

The interaction constants were calculated at an incident pump power below the saturation of the blueshift $\left(150 \mathrm{~W} \mathrm{~cm}^{-2}\right)$ and at the instant of highest total polariton density in momentum space. The $\mathbf{k}$ dependent blueshift $\Delta E_{L P}(\mathbf{k})$ is given by

$$
\Delta E_{L P}(\mathbf{k})=\sum_{\mathbf{k}^{\prime}} E_{\mathbf{k}, \mathbf{k}^{\prime}}^{\text {shift }}\left|\psi_{L P}\left(\mathbf{k}^{\prime}\right)\right|^{2},
$$

where $E_{\mathbf{k}, \mathbf{k}^{\prime}}^{\text {shift }}$ is the polariton-polariton interaction constant ${ }^{6}$. Substituting the expressions for each interaction mechanism individually (see Supplementary Information), we can obtain the exciton-exciton interaction constant as

$$
V_{X X}(\mathbf{k})=\frac{\Delta E_{L P}(\mathbf{k})}{2\left|X_{\mathbf{k}}\right|^{2} \sum_{\mathbf{k}^{\prime}}\left|X_{\mathbf{k}^{\prime}}\right|^{2}\left|\psi_{L P}\left(\mathbf{k}^{\prime}\right)\right|^{2}}
$$

and the saturation interaction constant as

$$
V_{S A T}(\mathbf{k})=\frac{\Delta E_{L P}(\mathbf{k})}{\left|C_{\mathbf{k}}\right|\left|X_{\mathbf{k}}\right| \sum_{\mathbf{k}^{\prime}}\left|X_{\mathbf{k}^{\prime}}\right|^{2}\left|\psi_{L P}\left(\mathbf{k}^{\prime}\right)\right|^{2}},
$$

where $\left|C_{\mathbf{k}}\right|$ and $\left|X_{\mathbf{k}}\right|$ are the Hopfield coefficients for the LP photon and exciton fractions, respectively. All the expressions and parameter values used in the calculations are listed in the Supplementary Information.

\section{REFERENCES}

* dario.ballarini@gmail.com 
† s.kena-cohen@polymtl.ca

1 Kin Fai Mak and Jie Shan, "Photonics and optoelectronics of 2 d semiconductor transition metal dichalcogenides," Nature Photonics 10, 216-226 (2016).

2 Marco Liscidini, Dario Gerace, Daniele Sanvitto, and Daniele Bajoni, "Guided bloch surface wave polaritons," Applied Physics Letters 98, 121118 (2011).

3 Giovanni Lerario, Alessandro Cannavale, Dario Ballarini, Lorenzo Dominici, Milena De Giorgi, Marco Liscidini, Dario Gerace, Daniele Sanvitto, and Giuseppe Gigli, "Room temperature bloch surface wave polaritons," Opt. Lett. 39, 2068-2071 (2014).

4 Stefano Pirotta, Maddalena Patrini, Marco Liscidini, Matteo Galli, Giacomo Dacarro, Giancarlo Canazza, Giorgio Guizzetti, Davide Comoretto, and Daniele Bajoni, "Strong coupling between excitons in organic semiconductors and bloch surface waves," Applied Physics Letters 104, 051111 (2014).

5 Giovanni Lerario, Dario Ballarini, Antonio Fieramosca, Alessandro Cannavale, Armando Genco, Federica Mangione, Salvatore Gambino, Lorenzo Dominici, Milena De Giorgi, Giuseppe Gigli, et al., "High-speed flow of interacting organic polaritons," Light: Science \& Applications 6, e16212 (2017).

${ }^{6}$ C Ciuti, P Schwendimann, and A Quattropani, "Theory of polariton parametric interactions in semiconductor microcavities," Semiconductor Science and Technology 18, S279 (2003).

7 A Amo, D Sanvitto, FP Laussy, D Ballarini, E Del Valle, MD Martin, A Lemaitre, J Bloch, DN Krizhanovskii, MS Skolnick, et al., "Collective fluid dynamics of a polariton condensate in a semiconductor microcavity," Nature 457, 291-295 (2009).

8 Alberto Amo, TCH Liew, Claire Adrados, Romuald Houdré, Elisabeth Giacobino, AV Kavokin, and A Bramati, "Exciton-polariton spin switches," Nature Photonics 4, 361 (2010).

9 T. Espinosa-Ortega and T. C. H. Liew, "Complete architecture of integrated photonic circuits based on and and not logic gates of exciton polaritons in semiconductor microcavities," Phys. Rev. B 87, 195305 (2013).

10 C Sturm, D Tanese, HS Nguyen, H Flayac, E Galopin, A Lemaître, I Sagnes, D Solnyshkov, A Amo, G Malpuech, et al., "All-optical phase modulation in a cavity-polariton mach-zehnder interferometer," Nature communications 5, 3278 (2014).

11 KS Daskalakis, SA Maier, Ray Murray, and Stéphane Kéna-Cohen, "Nonlinear interactions in an organic polariton condensate," Nature materials 13, 271-278 (2014).

12 Daniele Sanvitto and Stéphane Kéna-Cohen, "The road towards polaritonic devices," Nature materials 15, 10611073 (2016).

13 S Dufferwiel, S Schwarz, F Withers, AAP Trichet, F Li, M Sich, O Del Pozo-Zamudio, C Clark, A Nalitov, DD Solnyshkov, et al., "Exciton-polaritons in van der waals heterostructures embedded in tunable microcavities," Nature communications 6 (2015).

14 Xiaoze Liu, Tal Galfsky, Zheng Sun, Fengnian Xia, Erhchen Lin, Yi-Hsien Lee, Stéphane Kéna-Cohen, and Vinod M Menon, "Strong light-matter coupling in twodimensional atomic crystals," Nature Photonics 9, 30-34 (2015).

15 Lucas C Flatten, Zhengyu He, David M Coles, Aurelien AP Trichet, Alex W Powell, Robert A Taylor, Jamie H
Warner, and Jason M Smith, "Room-temperature excitonpolaritons with two-dimensional $\mathrm{WS}_{2}$," Scientific reports 6 (2016).

16 Shaojun Wang, Songlin Li, Thibault Chervy, Atef Shalabney, Stefano Azzini, Emanuele Orgiu, James A. Hutchison, Cyriaque Genet, Paolo Samorì, and Thomas W. Ebbesen, "Coherent coupling of $\mathrm{WS}_{2}$ monolayers with metallic photonic nanostructures at room temperature," Nano Letters 16, 4368-4374 (2016), pMID: 27266674.

17 Zheng Sun, Jie Gu, Areg Ghazaryan, Zav Shotan, Christopher R Considine, Michael Dollar, Biswanath Chakraborty, Xiaoze Liu, Pouyan Ghaemi, Stéphane Kéna-Cohen, et al., "Optical control of room-temperature valley polaritons," Nature Photonics 11, 491 (2017).

18 Wenjing Liu, Bumsu Lee, Carl H. Naylor, Ho-Seok Ee, Joohee Park, A. T. Charlie Johnson, and Ritesh Agarwal, "Strong exciton-plasmon coupling in $\mathrm{MoS}_{2}$ coupled with plasmonic lattice," Nano Letters 16, 1262-1269 (2016), pMID: 26784532.

19 Nils Lundt, Sebastian Klembt, Evgeniia Cherotchenko, Simon Betzold, Oliver Iff, Anton V Nalitov, Martin Klaas, Christof P Dietrich, Alexey V Kavokin, Sven Höfling, et al., "Room-temperature tamm-plasmon exciton-polaritons with a $\mathrm{WSe}_{2}$ monolayer," Nature communications 7 (2016).

20 A. Baas, J. Ph. Karr, H. Eleuch, and E. Giacobino, "Optical bistability in semiconductor microcavities," Phys. Rev. A 69, 023809 (2004).

21 Yilei Li, Alexey Chernikov, Xian Zhang, Albert Rigosi, Heather M. Hill, Arend M. van der Zande, Daniel A. Chenet, En-Min Shih, James Hone, and Tony F. Heinz, "Measurement of the optical dielectric function of monolayer transition-metal dichalcogenides: $\mathrm{MoS}_{2}, \mathrm{MoSe}_{2}$, $\mathrm{WS}_{2}$, and WSe 2 ," Phys. Rev. B 90, 205422 (2014).

${ }^{22}$ Humberto R. Gutiérrez, Nestor Perea-López, Ana Laura Elías, Ayse Berkdemir, Bei Wang, Ruitao Lv, Florentino López-Urías, Vincent H. Crespi, Humberto Terrones, and Mauricio Terrones, "Extraordinary room-temperature photoluminescence in triangular $\mathrm{WS}_{2}$ monolayers," Nano Letters 13, 3447-3454 (2013), pMID: 23194096.

23 Bairen Zhu, Xi Chen, and Xiaodong Cui, "Exciton binding energy of monolayer $\mathrm{WS}_{2}$," Scientific reports 5 (2015).

${ }^{24}$ Chunxiao Cong, Jingzhi Shang, Yanlong Wang, and Ting $\mathrm{Yu}$, "Optical properties of $2 \mathrm{~d}$ semiconductor $\mathrm{WS}_{2}$," Advanced Optical Materials 6, 1700767-n/a (2018), 1700767.

25 Long Yuan and Libai Huang, "Exciton dynamics and annihilation in $\mathrm{WS}_{2} 2 \mathrm{~d}$ semiconductors," Nanoscale 7, 74027408 (2015).

${ }^{26}$ K. S. Daskalakis, S. A. Maier, and S. Kéna-Cohen, "Spatial coherence and stability in a disordered organic polariton condensate," Phys. Rev. Lett. 115, 035301 (2015).

27 Giovanni Lerario, Antonio Fieramosca, Fábio Barachati, Dario Ballarini, Konstantinos S Daskalakis, Lorenzo Dominici, Milena De Giorgi, Stefan A Maier, Giuseppe Gigli, Stéphane Kéna-Cohen, et al., "Room-temperature superfluidity in a polariton condensate," Nature Physics (2017).

28 V. Shahnazaryan, I. Iorsh, I. A. Shelykh, and O. Kyriienko, "Exciton-exciton interaction in transition-metal dichalcogenide monolayers," Phys. Rev. B 96, 115409 (2017).

29 Angelo Angelini, Elsie Barakat, Peter Munzert, Luca Boarino, Natascia De Leo, Emanuele Enrico, Fabrizio Giorgis, Hans Peter Herzig, Candido Fabrizio Pirri, and Emiliano Descrovi, "Focusing and extraction of light me- 
diated by bloch surface waves," Scientific reports 4 (2014).

30 Yusuke Hoshi, Takashi Kuroda, Mitsuhiro Okada, Rai Moriya, Satoru Masubuchi, Kenji Watanabe, Takashi Taniguchi, Ryo Kitaura, and Tomoki Machida, "Suppression of exciton-exciton annihilation in tungsten disulfide monolayers encapsulated by hexagonal boron nitrides," Phys. Rev. B 95, 241403 (2017).

\section{ACKNOWLEDGMENTS}

SKC acknowledges support from the NSERC Discovery Grant Program and the Canada Research Chair in Hybrid and Molecular Photonics. FB acknowledges support from the FQRNT PBEEE scholarship program. LM acknowledges financial support from the NSERC Discovery Grant. Work at the City University of New York was supported by the National Science Foundation (NSF) under the EFRI 2-DARE program (EFMA-1542863) and the NSF-ECCS-1509551 grant. AF, DB and DS acknowledge the ERC "ElecOpteR" grant number 780757. SKC and
DS acknowledge support from the mixed Québec-Italy sub-commission for bilateral collaboration.

\section{AUTHOR CONTRIBUTIONS}

SKC conceived the project and FB designed the sample. The sample was fabricated by FB, SH, JG, BC under the supervision of LM, SKC and VM. Optical experiments were performed by FB, AF and DB. FB analyzed the data and wrote the manuscript. Numerical calculations were performed by FB and SKC. All authors contributed to revising the manuscript and analyzing the results. DB, DS and SKC coordinated the project.

\section{COMPETING FINANCIAL INTERESTS}

The authors declare no competing financial interests. 